\title{
Multiple benefits of physical activity during the Coronavirus pandemic
}

\section{Os múltiplos benefícios da atividade física durante a pandemia do coronavírus}

\begin{tabular}{l} 
AUTHOR'S \\
\hline James F. Sallis ${ }^{1,2}$ (D) \\
Michael Pratt ${ }^{1}$ (D) \\
1 Department of Family and Preventive Medicine, \\
MC0631. University of California San Diego. 9500 \\
Gilman Dr. La Jolla, California, USA. \\
2. Mary Mackillop Institute for Health Research, \\
Australian Catholic University. Level 5, 215 Spring \\
Street. Melbourne, Australia. \\
CORRESPONDING \\
\hline James F. Sallis \\
jsallis@health.ucsd.edu \\
Department of Family and Preventive Medi- \\
cine, MC0631. \\
University of California San Diego. 9500 \\
Gilman Dr. La Jolla, California, USA. \\
Zip code: $92093-0631$. \\
DOI
\end{tabular}

10.12820/rbafs. $25 \mathrm{e} 0112$

\section{(cc) BY}

This work is licensed under a Creative Commons Attribution 4.0 International License.
Physical activity is one of the strongest forces for good health. Physical activity helps prevent and/or treat many physical and mental health conditions by improving functioning of numerous physiological systems ${ }^{1}$. In this piece we explain how harnessing the salutogenic power of physical activity could help ease the consequences of the coronavirus pandemic in six ways. This commentary is an expanded version of a blog that was distributed to leaders in physical activity and public health in early April 2020 to bring awareness of the relevance of physical activity during the coronavirus pandemic. Though it is not clear whether the blog has contributed to any increase in priority for physical activity in the pandemic response, it has been posted online in several languages ${ }^{2}$ and adapted for an online news source in India ${ }^{3}$. The current version adds two more likely benefits to the original four, provides ideas on promoting physical activity more equitably during the pandemic, and offers suggestions for planning for more physical activity as cities and countries relax shelter-in-place restrictions. This commentary is motivated by a perceived urgency to add physical activity's benefits to efforts to manage the current public health crisis, so we are grateful for the opportunity to include this commentary in the timely release of this Special Issue of the Brazilian Journal of Physical Activity and Health on the coronavirus pandemic.

We begin with identification of six likely benefits of physical activity with direct relevance to the pandemic, referencing just a sample of supporting literature, mainly review papers. First, physical activity has potential to reduce the severity of COVID-19 infections. This is related to what happens in the lungs during an infection. The immune system detects the invading virus in the lungs and attacks it. The conflict between the virus and immune cells creates inflammation. That inflammation causes damage to lung tissue that interferes with gas exchange and can become severe enough to require medical interventions, such as mechanical ventilators ${ }^{4}$.

Physical activity has favorable effects on these physiological mechanisms. During physical activity, muscles produce compounds that improve functioning of the immune system and reduce inflammation ${ }^{5}$. Thus, physical activity strengthens the two biological processes that react to the infection. Although studies of the effects of exercise have not been conducted with COVID-19 patients, the effects of exercise on immunity, inflammation $^{6-10}$, and viral respiratory infections ${ }^{11}$ are well documented. Because muscles make up 30-40\% of body weight, they can be a powerful ally in fighting the impact of infection, but only when the muscles are being used. Moderate-intensity physical activities, like walking, have the best impact, 
but extreme vigorous exercise, like running a marathon, temporarily reduces immune function ${ }^{11}$. The potential for increased physical activity to reduce the number of infected people who require hospitalization and use of ventilators could help reduce the extent to which health care systems become overwhelmed by cases of severe infection. Reduced severity of infections should also lessen health care costs created by the pandemic, as well as ease suffering.

Second, physical activity is effective for both preventing and treating hypertension, obesity, heart diseases, diabetes, and eight types of cancers ${ }^{1}$, all of which increase risk of severe illness and death among those infected with the coronavirus. Though physical activity is widely recommended by health authorities throughout the world, efforts to promote active lifestyles are minimal ${ }^{12}$. It makes sense now to encourage and assist people, especially those with chronic conditions, to be moderately active prior to any potential exposure to viral infection, so as to reduce severity of illness if infection should occur. There is specific evidence that physical activity's immunity and inflammation benefits are seen in most of the high risk groups listed above ${ }^{13}$. Because each session of physical activity has acute effects on immune functioning and inflammation ${ }^{5}$, similar to taking a medication daily, people can reduce their risk of severe viral infections and their risk from multiple chronic diseases by simply taking a walk every day. It is not too late in the pandemic for people to benefit from modest increases in their physical activity.

Third, symptoms of stress are virtually certain to increase as the pandemic continues, due to health threats, job loss, reduced income, and isolation from social contact. Fortunately, being physically active has important mental health benefits, and encouraging people to be active could help them cope with ongoing stress and avoid psychological ill-health. Each session of physical activity reduces symptoms of depression and anxiety ${ }^{14}$, so being active every day can be a partial antidote to the stress of the pandemic. For people already feeling distress, being active has similar effectiveness as medications and psychotherapy $^{1}$. The most common physical activity is walking, which is free, accessible to most people of all ages $^{15}$, and lends itself well to physical distancing.

Fourth, the body's response to psychological stress creates imbalances between cortisol and other hormones that negatively affect the immune system and inflammation. Thus, psychological stress affects the underlying biological processes of COVID-19, and re- storing cortisol balance is another mechanism by which physical activity benefits immunity and inflammation. The most effective strategies for improving cortisol balance are physical activity and stress management ${ }^{16}$. Because older people have disrupted cortisol physiology and weaker immune systems ${ }^{16}$, physical activity may be particularly important for this large population at high risk for COVID-19.

Fifth, there may be a protective effect on lung function among physically active and fit individuals. Pulmonary function does not generally respond to exercise training. This may seem counter intuitive, but healthy individuals at all ages have sufficient reserve capacity for ventilation and pulmonary gas exchange so that respiratory function is not a limiting factor for exercise $^{17}$. However, endurance exercise induces production of antioxidants that confer protection against oxidative stress and may specifically prevent or reduce the severity of acute respiratory distress syndrome (ARDS). ARDS is a serious and common outcome among those hospitalized with COVID-19, and patients who have been regularly active may have a degree of protection against $\mathrm{ARDS}^{18}$.

Sixth, physical activity may be able to play a role in what is considered the ultimate solution to the coronavirus pandemic. The expectation is that eventual production of an effective vaccine that is delivered worldwide will allow resumption of relatively "normal" economic and social activities ${ }^{19}$. Both acute and chronic physical activity have been shown repeatedly to improve the immune response to vaccines ${ }^{20}$, with longer-term exercise resulting in more people with antibody levels sufficient to protect from infections ${ }^{13}$. In one study, older adults were assigned to either moderate-intensity aerobic exercise or a flexibility and balance intervention that occurred before and after an influenza vaccination. Participants in the aerobic group were $30-100 \%$ more likely than the flexibility group to attain sufficient antibodies to protect them from three strains of influenza virus, six months after the vaccination ${ }^{21}$. The vaccination-enhancement effect may be especially important for older adults who have reduced responses to vaccines ${ }^{20}$ and are at much higher risk for severe forms of COVID-19.

COVID-19 has exposed underlying weaknesses in public health preparedness and health equity, especially in the United States. Current data from the U.S. Centers for Disease Control and Prevention (CDC) suggest a disproportionate burden of COVID-19 re- 
lated illness and death among racial and ethnic minority groups ${ }^{22}$. Many factors contribute to this disparity, including poor access to health care, housing and food insecurity, poor access to safe open spaces for being physically active while maintaining safe physical distancing, lack of paid sick leave, an over-representation in jails and prisons that carry the increased risks of congregate living, and poorer underlying health due to chronic diseases. Though COVID-19 impacts everyone, it hits hardest those who have the fewest resources and face ongoing discrimination and stress. We must place special emphasis on providing supportive programs and ensuring access to safe, attractive places for outdoor physical activity for persons who face socio-economic challenges. Similarly, needs of people with mobility limitations should be taken into consideration during planning. Otherwise, poorly-designed efforts to promote physical activity may further exacerbate societal and health inequities.

The most important actions at the time of this writing are to reduce spread of the coronavirus through physical distancing, frequent hand washing, and avoiding touching the face. But, due to its multiple benefits, physical activity should not be an afterthought during this pandemic. Being active should be a key recommendation. People need to know about actions they can take themselves to help reduce the risk of severe infections and stressful reactions to the pandemic. Worldwide, about $23 \%$ of men and $32 \%$ of women are at risk ${ }^{23}$ because they do not meet physical activity guidelines ${ }^{24}$. We recommend immediately beginning studies on the impact of regular physical activity on the severity of illness among people infected by the coronavirus. One important study would be to assess physical activity at the time of COVID-19 testing, then follow-up patients to determine whether activity reduces severity of the infection and enhances survival. Similar studies conducted in multiple countries would be valuable. But action does not need to wait for a study. Physical activity is already almost universally recommended ${ }^{24}$, and there is much evidence that physical activity could contribute to reducing the severity of COVID-19 illness, enhancing quality of life before and after infection, and enhancing efficacy of eventual vaccines. What is missing is a concerted and resourced effort to implement some of the many evidence-based physical activity interventions ${ }^{12,25}$.

We have been encouraged by media stories and online posts from citizens encouraging people to be active during the current crisis $^{26}$. We are pleased that, so far, exercising has been considered an "essential activity" as part of orders for sheltering in place in most countries. But the potential for physical activity to reduce the severity of COVID-19 infection is not a communication goal in any country to our knowledge. Although exercise outdoors is permitted in most countries during shelter-in-place orders, closures of parks, trails, gyms, and beaches make it more difficult for people to be active. Popular places for physical activity should be retained as much as possible, but it may be necessary to monitor outdoor locations to ensure people maintain safe physical distancing. Any kind of enjoyable moderate activity, indoors or outdoors, is great for mind and body, but people, including decision makers in each country, need to understand the many important roles physical activity can play in reducing their risk of severe infection and maintaining quality of life.

At the time of this writing, many countries are under shelter-in-place orders, but countries with the earliest outbreaks are beginning to loosen restrictions. Because of the risks of re-occurrence of outbreaks, as was seen in the 1918 influenza pandemic ${ }^{27}$, it is likely that after opening up and experiencing more outbreaks, restrictions will be imposed again. This extended process with varying levels of restrictions presents opportunities to take advantage of the power of physical activity.

Physical activity organizations and leaders should develop plans within each country, and with the WHO, to make physical activity education and promotion a public health goal during and after the pandemic. Physical activity organizations and experts should volunteer for pandemic planning groups so they educate decision makers about how physical activity can make positive impacts on the pandemic and influence recommendations and policies. Here are several actions that may be helpful as part of shelter-in-place orders or when economic and social activities resume.

Official decision-making groups or health organizations acting independently should educate populations about the six pandemic-related benefits described above. Some of the messaging should be tailored for the high-risk groups of older adults and those with pre-existing conditions. Health care providers should be provided with information and tools that can help their patients start or maintain regular physical activity. Exercise Is Medicine is an international initiative that already has many relevant materials ${ }^{28}$.

In many areas, health clubs, parks, trails, and beach- 
es have been closed, specifically because they were popular places for physical activity. These closures may be counter-productive if they have the effect of decreasing physical activity. Thus, plans are needed to safely reopen these important physical activity environments in ways that do not contribute to promoting spread of the virus $^{29}$. Education about the need for physical distancing, monitoring of crowding, limiting the number of users at any one time, rearranging or temporarily removing workout equipment, and enforcement of safety protocols will likely be needed. Physical activity experts should be involved in developing such protocols.

Although children and adolescents are at low risk of severe coronavirus infections, closing schools, playgrounds, and parks is likely to reduce their physical activity and harm their physical and psychological health ${ }^{1}$. Physical educators, parks professionals, pediatricians, and others should become involved in decision making about how to help children be active during shelter-in-place orders and how to reopen these opportunities for physical activity in a safe manner. For example, modified, distanced physical education lessons are needed. Guidelines for modifications to, and monitoring of, playgrounds and parks will be needed until widespread vaccination is achieved.

Rather than close popular places for physical activity, an alternative strategy is to provide more space for physical activity, so it is easier to maintain safe distances. Several groups are promoting a major expansion of "open streets" programs that close streets to traffic but allow walkers, joggers, bicyclists, and non-motorized wheelers to use the streets ${ }^{30-32}$. This could be an equitable and low-cost solution for providing physical activity opportunities if open streets become a regular feature in all neighborhoods so people do not have to travel far to access them. Establishing open streets during shelter-in-place orders could generate demand, experience, and protocols that could be applied to continuing them as society reopens.

Planning responses to the coronavirus pandemic that make use of the salutogenic power of physical activity will require effective advocacy to get physical activity experts in the room where decisions are being made, and creative thinking to generate feasible and promising solutions to problems the field has not encountered before. Global physical activity promotion initiatives ${ }^{33}$ and national physical activity plans $^{34}$ are existing frameworks that probably need to be revised in the wake of the current pandemic. Simultaneously, the physical activity-related disciplines need to quickly begin research to develop evidence that guides better policies, programs, and recommendations as we progress through the phases of the pandemic. What we learn during this pandemic can be used to ensure the benefits of physical activity are fully realized in future crises.

\section{Conflicts of interest}

The authors declare no conflict of interest.

\section{Authors' contributions}

Sallis JF conceptualized the paper, wrote the first draft, reviewed subsequent drafts, and approved the final manuscript. Pratt $\mathrm{M}$ drafted multiple sections, reviewed all drafts, and approved the final manuscript.

\section{References}

1. Powell KE, AC King, DM Buchner, WW Campbell, L DiPietro, KI Erickson, et al. The scientific foundation for the Physical Activity Guidelines for Americans, 2nd Edition. J Phys Act Health. 2018:1-11.

2. Sallis J, Pratt M. A call to action: Physical activity and COVID-19. [Accessed 2020 April 24]. Available from: Blog in English: https://www.exerciseismedicine.org/support_ page.php/stories/?b=896. Blog in Spanish: https://rafapana. org/la-actividad-fisica-puede-ser-util-en-la-pandemia-decoronavirus/.

3. Adlahka D, Sallis JF, Pratt M. COVID-19 and India's chronic disease burden: How exercise can be beneficial. The News Minute (India). [Accessed 2020 April 24]. Available from: https://www.thenewsminute.com/article/covid-19and-india-s-chronic-disease-burden-how-exercise-can-bebeneficial-122684.

4. Shi Y, Wang Y, Shao C, Huang J, Gan J, Huang X, Bucci E, Piacentini M, Ippolito G, Melino G. COVID-19 infection: the perspectives on immune responses. Cell Death Differ. 2020;27:1451-4.

5. Hojman P. Exercise protects from cancer through regulation of immune function and inflammation. Biochem Soc Trans. 2017;45(4):905-11.

6. Campbell JP, Turner JE. Debunking the myth of exerciseinduced immune suppression: redefining the impact of exercise on immunological health across the lifespan. Front Immunol. 2018;9:648.

7. Jones AW, Davison G. Exercise, immunity, and illness. In Muscle and Exercise Physiology. 2019:317-44. Academic Press.

8. Lee DH, de Rezende LFM, Eluf-Neto J, Wu K, Tabung FK, Giovannucci EL. Association of type and intensity of physical activity with plasma biomarkers of inflammation and insulin response. Internat J Cancer. 2019;145(2):360-9.

9. Meneses-Echávez JF, Correa-Bautista JE, González-Jiménez E, Río-Valle JS, Elkins MR, Lobelo F, Ramírez-Vélez R. The effect of exercise training on mediators of inflammation in breast cancer survivors: a systematic review with meta-analysis. Cancer Epidemiol Prev Biomark. 2016;25(7):1009-17.

10. Miles MP, Wilson S, Yeoman CJ. Physical activity and inflammation phenotype conversion. J Clin Exerc Physiol. 2019;8(2):64-73. 
11. Nieman DC, LM Wentz. The compelling link between physical activity and the body's defense system. J Sport Health Sci. 2019;8(3):201-17.

12. Reis RS, Salvo D, Ogilvie D, Lambert EV, Goenka S, Brownson RC. \& Lancet Physical Activity Series 2 Executive Committee. Scaling up physical activity interventions worldwide: stepping up to larger and smarter approaches to get people moving. Lancet. 2016;388(10051):1337-48.

13. Simpson RJ, Kunz H, Agha N, Graff R. Exercise and the regulation of immune functions. Progr Mol Biol Transl Sci. 2015;135:355-80.

14. Basso JC, Suzuki W.A. The effects of acute exercise on mood, cognition, neurophysiology, and neurochemical pathways: A review. Brain Plast. 2017;2(2):127-52.

15. Centers for Disease Control and Prevention. Active People, Healthy Nation, 2018. [Accessed 2020 April 24]. Available from: https:/www.cdc.gov/physicalactivity/ activepeoplehealthynation/about-active-people-healthynation.html.

16. Adam EK, Quinn ME, Tavernier R, McQuillan MT, Dahlke KA, Gilbert KE. Diurnal cortisol slopes and mental and physical health outcomes: A systematic review and metaanalysis. Psychoneuroendocrinol 2017;83:25-41.

17. Roman MA, Rossiter HB, Casaburi R. Exercise, ageing and the lung. Eur Respir J. 2016;48(5):1471-86.

18. Yan Z, Spaulding HR. Extracellular superoxide dismutase, a molecular transducer of health benefits of exercise. Redox Biol. 2020;19;32:101508.

19. Lichfield, G. This is what it will take to get us back outside. MIT Technology Review. [Accessed 2020 April 24]. Available from: https://www.technologyreview. com/2020/04/12/999117/blueprint-what-it-will-take-tolive-in-a-world-with-covid-19/.

20. Pascoe AR, Singh MAF, Edwards KM. The effects of exercise on vaccination responses: a review of chronic and acute exercise interventions in humans. Brain Behav Immun. 2014;39:33-41.

21. Woods JA, Keylock KT, Lowder T, Vieira VJ, Zelkovich W, Dumich S, Colantuano K, Lyons K, Leifheit K, Cook M, Chapman-Novakofski K. Cardiovascular exercise training extends influenza vaccine seroprotection in sedentary older adults: the immune function intervention trial. J Am Geriatr Soc. 2009;57(12):2183-91.

22. Garg S, Kim L, Whitaker M, et al. Hospitalization rates and characteristics of patients hospitalized with laboratoryconfirmed coronavirus disease 2019 - COVID-NET, 14 States, March 1-30, 2020. Morb Mortal Wkly Rep. 2020;69:458-64.
23. Guthold R, Stevens GA, Riley LM, Bull FC. Worldwide trends in insufficient physical activity from 2001 to 2016: a pooled analysis of 358 population-based surveys with 1. 9 million participants. Lancet Global Health. 2018 Oct 1;6(10):e1077-86.

24. World Health Organization, 2019. [Accessed 2020 April 24]. Available from: https://www.who.int/dietphysicalactivity/ factsheet_recommendations/en/,

25. King AC, MC Whitt-Glover, DX Marquez, MP Buman, MA Napolitano, J Jakicic, et al. Physical activity promotion: Highlights from the 2018 Physical Activity Guidelines Advisory Committee systematic review. Med Sci Sports Exerc. 2019;51(6):1340-53.

26. Koren, M. The healthiest way to sweat out a pandemic. The Atlantic. [Accessed 2020 April 24]. Available from: https:// www.theatlantic.com/health/archive/2020/03/coronavirusgyms-exercise-social-distancing/608278/.

27. Short KR, Kedzierska K, van de Sandt CE. Back to the future: Lessons learned from the 1918 influenza pandemic. Front Cell Infect Microbiol. 2018;8:343.

28. Exercise Is Medicine. Available from: https://www. exerciseismedicine.org/

29. Honey-Roses J, Anguelovski I, Bohigas J, Chireh V, Daher C, Konijnendijk C, Litt J, Mawani V, McCall M, Orellana A, Oscilowicz E. The impact of COVID-19 on public space: A review of the emerging questions. 2020. [Accessed 2020 April 24]. Available from: https://doi.org/10.31219/osf.io/ rf7xa . https://osf.io/rf7xa/.

30. 8-80 Cities. [Accessed 2020 April 24]. Available from: https://www.880cities.org/.

31. National Complete Streets Coalition. [Accessed 2020 April 24]. Available from: https://smartgrowthamerica.org/closedcities-should-open-streets/.

32. Rails to Trails Conservancy. [Accessed 2020 April 24]. Available from: https:/www.railstotrails.org/ trailblog/2020/march/27/closing-streets-to-create-spacefor-walking-and-biking/.

33. World Health Organization, 2018. [Accessed 2020 April 24]. Available from: https://www.who.int/publicationsdetail/global-action-plan-on-physical-activity2018\%E2\%80\%932030.

34. Bornstein DB, Pate RR, Pratt M. A review of the national physical activity plans of six countries. J Phys Act Health. 2009;6(s2):S245-64.

Received: 26/05/2020

Approved: 27/05/2020 